\title{
Research on the Design of Disaster Prevention Plan for 22km Ultra-long Highway Tunnel with Multiple Intersections
}

\author{
Xuan Zhang ${ }^{1}$, Xuefeng $\mathrm{Li}^{1 *}$, and Xialin $\mathrm{Liu}^{2}$ \\ ${ }^{1}$ Research Institute of Highway, Ministry of Transport, Beijing 100088, China \\ ${ }^{2}$ CCCC Second Highway Survey, Design and Research Institute Co., Ltd, Wuhan, Hubei 430056, China
}

\begin{abstract}
The Tianshan Shengli Tunnel is currently the longest high-altitude highway tunnel. In order to ensure the safe operation of the tunnel, the SES software is used to investigate the wind speed laws in the tunnel, and the tunnel is divided into 16 disaster prevention sections based on the shaft and critical wind speed. The principles of disaster prevention and rescue of Tianshan Shengli Highway Tunnel are given, and the emergency treatment process of tunnel disaster prevention and rescue is given. The disaster prevention and rescue plan of Tianshan Shengli Tunnel from fire occurrence, personnel and vehicle evacuation, and fire treatment are discussed in detail.
\end{abstract}

\section{Introduction}

Highway tunnels have narrow space and limited safe evacuation exits. After a fire occurs, the fire fighting route is single, resulting in the detention of people and vehicles. The toxic gas and smoke generated by the fire will pose a serious threat to the people in the tunnel ${ }^{[1,2]}$. In order to ensure the orderly development of evacuation and rescue work, it is necessary to establish a scientific and effective disaster prevention plan. The establishment of a disaster prevention plan can reduce the casualties that may be caused by the fire, reduce property losses, and improve the operation and management level of the tunnel ${ }^{[3]}$.

Wang Mingnian et al. ${ }^{[4]}$ studied the disaster prevention and rescue of the Qinling Zhongnanshan Highway Tunnel and found that the fire control in the tunnel includes three parts: system linkage, traffic evacuation, and rescue and fire extinguishing. Li Guohui ${ }^{[5]}$ analyzed the emergency rescue of the Erlangshan Tunnel on the Sichuan-Tibet Highway and obtained the basic theories of the organization and command, force mobilization, combat deployment and disposal procedures of the tunnel emergency rescue. Zhou Qing et al. ${ }^{[6]}$ summarized the basic principles of tunnel disaster prevention and rescue, established a set of tunnel disaster prevention and rescue management system, and analyzed the process of tunnel disaster prevention and rescue system and the factors affecting fire evacuation and rescue.

The Tianshan Shengli Highway Tunnel is located on the southwest side of Urumqi, Xinjiang. It is a two-way four-lane road with a length of more than $22 \mathrm{~km}$ and an altitude of $2772 \mathrm{~m}$. It is an ultra-long high-altitude highway tunnel. The straight-line distance from the entrance to Urumqi is about $92 \mathrm{~km}$, and the straight-line distance from the exit to Hejing County is about $76 \mathrm{~km}$. The starting and ending pile numbers of the left line of the tunnel are $\mathrm{ZK} 75+660 \sim \mathrm{ZK} 97+790$, the length is $22130 \mathrm{~m}$, the maximum buried depth is about $1115 \mathrm{~m}$; the starting and ending pile numbers of the right line are YK75+790 YK97+821.7, the length is $22031.7 \mathrm{~m}$, the maximum buried depth is about approximately $1122.2 \mathrm{~m}$. The tunnel is composed of two lines on the left and right plus an intermediate service tunnel, with 3 vertical, 118 horizontal, and 4 connecting passages for fan rooms, and there are 560 intersections.

Based on the characteristics of the tunnel, the disaster prevention and rescue of the Tianshan Shengli Highway Tunnel is faced with the difficulties of ventilation, smoke control, personnel escape and rescue. Therefore, it is necessary to conduct an in-depth study on the disaster prevention zone and rescue plan of the Tianshan Shengli Tunnel to provide reference for the disaster prevention and rescue design of the tunnel.

\section{Disaster prevention and rescue zone division}

When a fire occurs, the direction of the wind flow in the tunnel is the same as the direction of the traffic. If the people upstream of the fire source cannot evacuate to the non-fire tunnel through the vehicle passing, they must get off the vehicle and evacuate through crosswalk. Vehicles downstream of the fire source will quickly drive away from the tunnel exit. Therefore, in the event of a fire, the downstream shaft closest to the fire source exhausts air, and the other shafts continuously supply air to the tunnel, so that the toxic gas and smoke in the tunnel can be discharged, ensuring that the fire smoke is controlled between the fire source and the closest exhaust outlet

\footnotetext{
* Corresponding author: 1xfsure@163.com
} 
downstream of the fire source. Guidelines for Ventilation Design of Highway Tunnels stipulates that the minimum fire critical wind speed is $2 \mathrm{~m} / \mathrm{s}$ [7], where the fire critical wind speed for smoke control is $2 \mathrm{~m} / \mathrm{s}$. The specific schematic diagram of tobacco control is shown in Figure 1 .

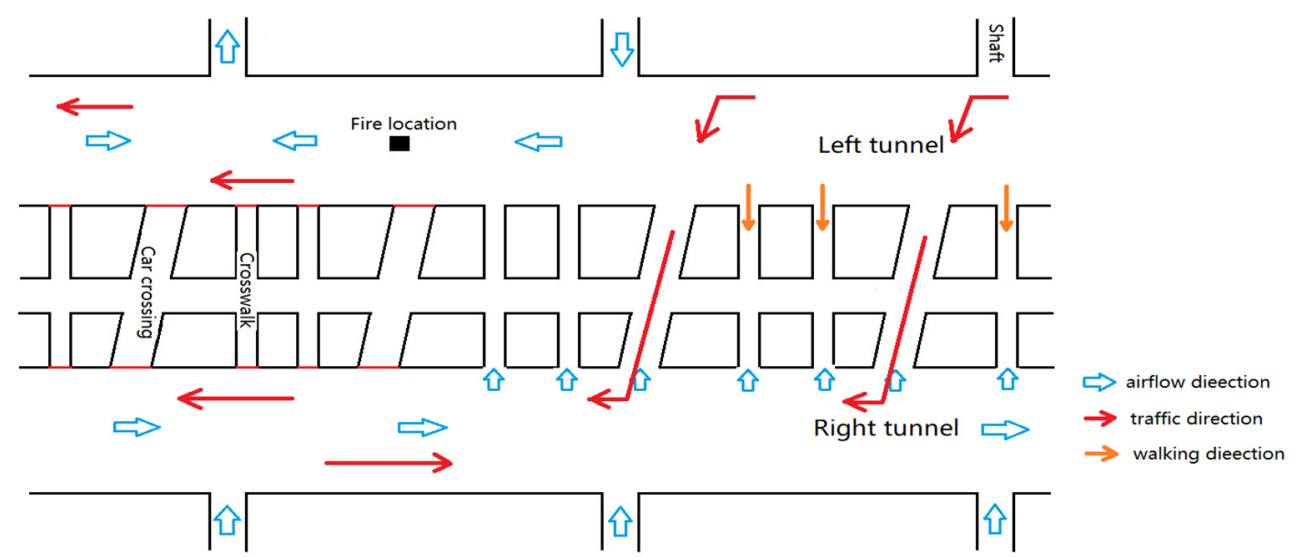

Figure 1. Schematic diagram of tobacco control

Taking the tunnel shaft as the boundary, the left and right tunnels are each divided into 5 pre-zones, a total of 10 pre-zones. Use SES software to simulate the exhaust ventilation under each pre-zone fire. The principle is as follows: Take a wind network composed of 5 edges and 4

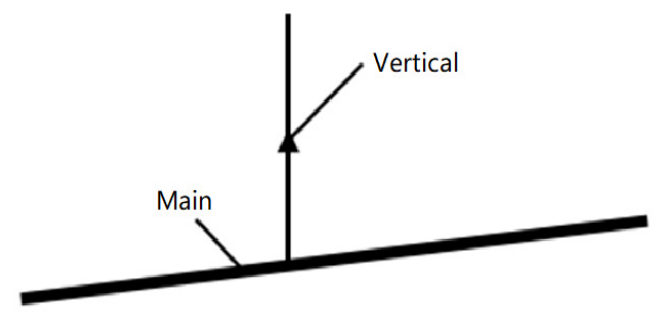

Figure 2. Schematic diagram of ventilation system

The basic laws of wind flow in the tunnel ventilation network are as follows:

(1) The law of air volume balance for a node in the ventilation network, according to the continuity equation, the amount of air flowing into the node should be equal to the amount of air flowing out of the node, namely:

$$
\sum Q_{i}=0
$$

In the formula: $\mathrm{Qi} \_$The amount of air flowing into or out of a node or mesh, flowing into a positive value, and flowing out of a negative value.

(2) The law of wind pressure balance

The algebraic sum of wind pressure at any mesh in the wind network (the wind pressure of the wind flow in the clockwise direction is positive, and the negative value in the counterclockwise direction) should be equal to zero. The so-called non-pressure source mesh refers to a loop where the mesh does not have the effect of natural wind pressure or fan or traffic ventilation. The wind pressure balance law is:

$$
\sum \Delta P_{i}=0
$$

In the formula: $\mathrm{Pi}$ the biased wind pressure in the mesh, which is negative and positive, counterclockwise. nodes as an example, where the arrow indicates the direction of wind flow, and the dashed line is the added natural wind pressure edge. The ventilation schematic diagram and network diagram of the ventilation tunnel are shown in Figure 2 and Figure 3.

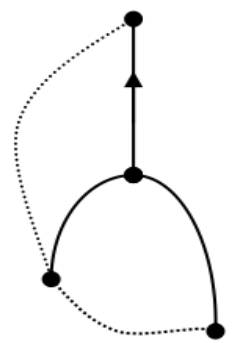

Figure 3. Ventilation network diagram

The pressure source mesh refers to the function of the mesh natural wind pressure exhaust or fan or traffic ventilation or air supply. The wind pressure algorithm is:

$$
\sum \triangle P_{i}-\left(\sum H_{\text {fan }}+\sum H_{\text {self }}+\sum \triangle P_{t}\right)=0
$$

In the formula: $\mathrm{H}_{\mathrm{fan}} \longrightarrow$ wind pressure of the fan in the mesh, clockwise to be positive, counterclockwise to be negative;

$\mathrm{H}_{\text {self }} \longrightarrow$ wind pressure in the mesh, the sign of the sign is the same as above;

$\triangle \mathrm{Pt} \longrightarrow$ Traffic ventilation capacity, the sign of the sign is the same as above;

(3) The law of resistance

The wind flow in the tunnel wind path is almost all stable turbulent flow, so the ventilation resistance is proportional to the square of the air volume.

$$
\triangle P=R Q_{2}
$$

In the formula: $\triangle \mathrm{P}$ _ is the ventilation pressure or ventilation resistance on the wind road, $\mathrm{Pa}$;

$\mathrm{R}$ — is the wind resistance on the wind road, $\mathrm{kg} / \mathrm{m} 7$;

$\mathrm{Q}$ _ is the air volume passing through the wind road, $\mathrm{m} 3 / \mathrm{s}$.

On the basis of the conservation of air volume at the nodes of the ventilation network, the critical smoke 
control wind speed of $2 \mathrm{~m} / \mathrm{s}$ is used as the partition boundary to determine the economic opening number of the fans and the detailed partition of the pre-partition. The
Tianshan Shengli Tunnel is divided into 16 disaster prevention zones. The overall disaster prevention zone division diagram of the tunnel is shown in Figure 4.

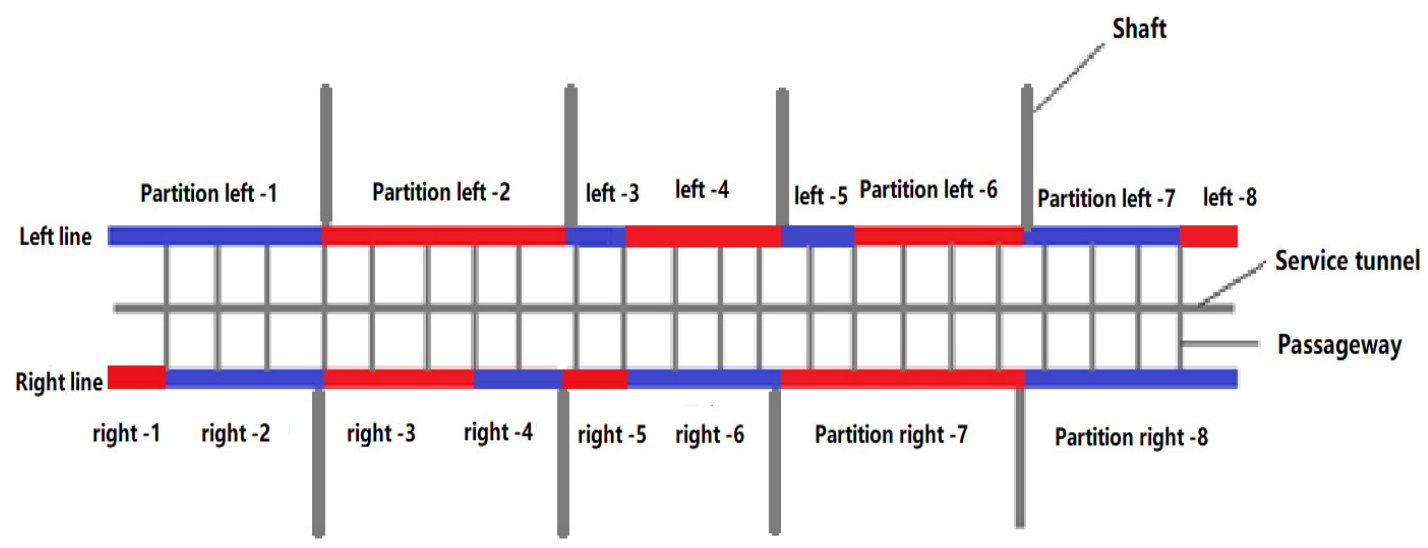

Figure 4. The overall disaster prevention zone of the tunnel

\section{Disaster prevention and rescue plan control process}

The disaster prevention and rescue plan of the Tianshan Shengli Tunnel should adhere to the basic ideas of prevention, alarm, monitoring, evacuation, rescue and fire fighting, and implement the general principle of "peopleoriented, prevention-oriented, and combination of prevention and fire fighting ", and ensure the safety of personnel as the primary principle, and establish a safety precaution system for fire warning, rescue, evacuation of personnel and vehicles, and fire extinguishing. On the basis of zoning, different disaster prevention plans are established, and corresponding disaster prevention measures are initiated according to the different locations of the fire.

When a fire occurs in the tunnel, measures shall be taken at the initial stage of the fire to evacuate people and vehicles in the fire tunnel in time to reduce the further spread of the fire. The control sequence in case of fire is shown in Figure 5.

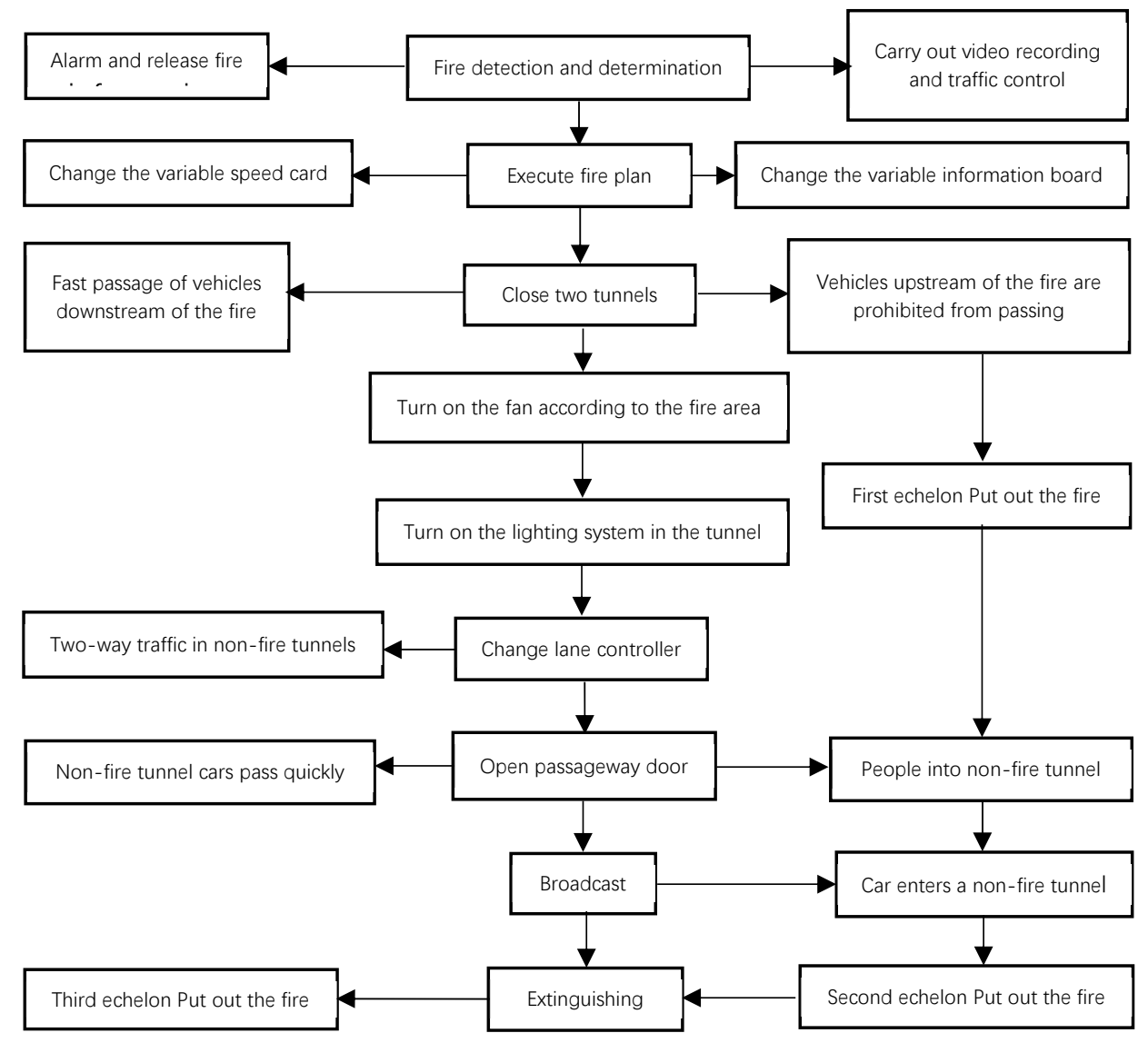

Figure 5. Control sequence in case of fire 
The detailed control sequence in case of fire is as follows:

(1) After the fire is confirmed, contact the professional staff of the corresponding unit to rush to the scene for evacuation, rescue and fire fighting, and implement the disaster prevention and rescue plan.

(2) Close the tunnel entrance. The traffic light at the tunnel entrance is red, indicating that entry is prohibited. The prompt message of the variable information board becomes "Tunnel fire, no entry", and the speed of the variable speed board becomes 0 . At the same time, the fire information was released, the drivers and passengers were called to prepare to escape through the broadcast in the tunnel, and vehicles outside the tunnel were prohibited from entering the tunnel through the broadcast outside the tunnel.

(3) Determine the number and direction of the fan opening according to the fire location. The direction of the upstream ventilation of the fire is the same as the driving direction, and the wind speed is greater than $2 \mathrm{~m} / \mathrm{s}$; the smoke generated by the fire is removed through the vertical shaft closest to the fire area to prevent the smoke from flowing back. Turn on the fan in the non-fire tunnel to prevent the smoke from spreading to the non-fire tunnel through the cross passage. Turn on all the lighting systems in the tunnel to facilitate fire fighting and personnel escape.
(4) Open all the cross passage doors upstream of the fire, the broadcast system will broadcast, and the drivers and passengers in the upstream will escape to the non-fire tunnel through the pedestrian and vehicular cross passage. The non-fire tunnel becomes a two-way lane, and the driving speed is limited to $30 \mathrm{~km} / \mathrm{h}$, and overtaking is strictly prohibited. Vehicles downstream of the fire passed orderly and quickly.

(5) After the evacuation of personnel is completed, organize professionals to put out the fire.

\section{Disaster prevention and rescue plans}

The smoke generated by the fire is exhausted through the vertical shaft closest to the fire area downstream to prevent the smoke from flowing back. Turn on the fan in the nonfire tunnel to prevent the smoke from spreading to the nonfire tunnel through the cross passage. The drivers and passengers in the upper reaches escaped to the non-fire tunnel through pedestrian and vehicular cross passage. The non-fire tunnel becomes a two-way lane, and vehicles downstream of the fire pass in an orderly and fast manner. Take the Tianshan Shengli Tunnel Zone Left-4 as an example, the schematic diagram of disaster prevention and rescue plan is shown in Figure 6.

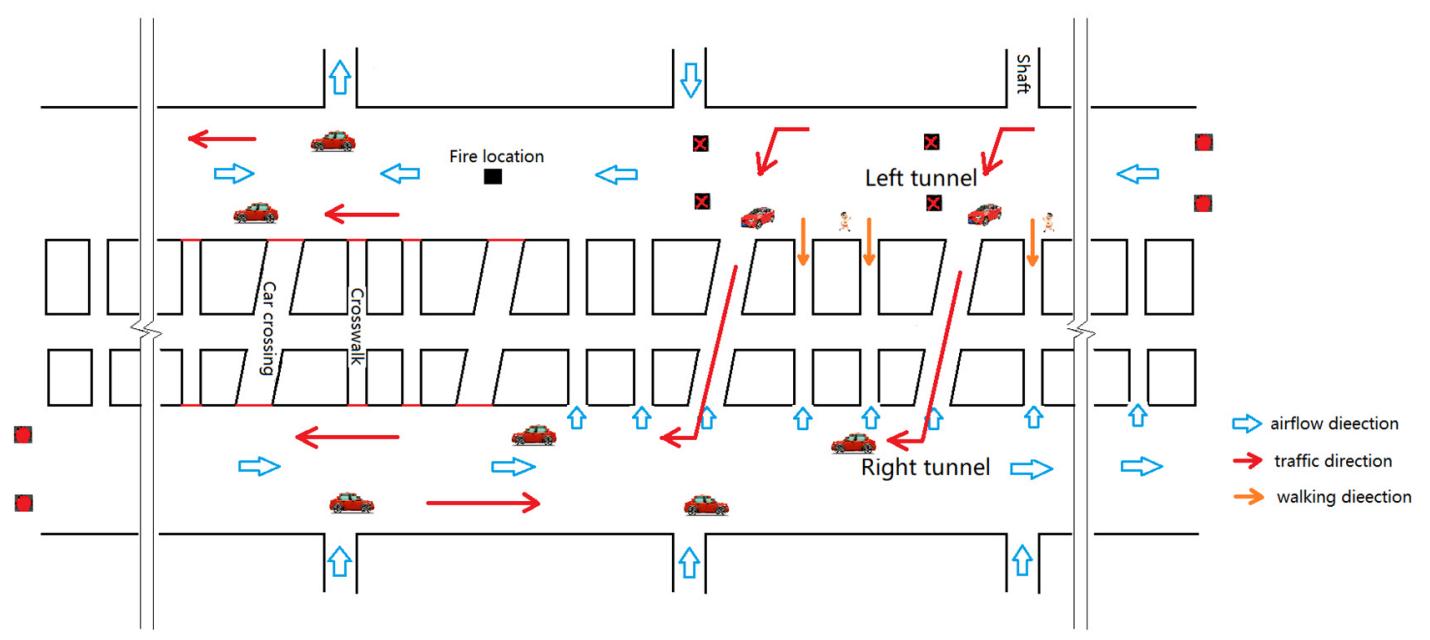

Figure 6. Disaster prevention zone left-4 plan diagram under fire

\section{Conclusion}

Through the research on the disaster prevention plan of the Tianshan Shengli Tunnel, the following main conclusions are obtained:

(1) Based on the location of the shaft and the critical wind speed, the Tianshan Shengli Tunnel is divided into 16 disaster prevention zones;

(2) Proposed the disaster prevention and rescue process of Tianshan Shengli Tunnel in combination with the mechanism system, wind flow direction and electromechanical system;

(3) Considering the disaster prevention zone and characteristics, the disaster prevention plan design of the Tianshan Shengli Tunnel is given, including the ventilation plan and the driving plan.

\section{References}

1. X. Zhou, X. Zhao, Y. Ding, Z. Zheng. Numerical studies on the critical Velocity and smoke distribution affected by tunnel cross-section geometry in tunnel fire. J Guangzhou Univ. 5,54(2006)

2. W. Liu, C. Zeng, Y. Yu, H. Huang. Study on fire preventing technology for subsea tunnel of Xiamen east passage// Proceedings of the 2006 International Conference on Road Tunnel Operation Management and Safety. Chongqing: Chongqing University Press,(2006)

3. L. Yu. Research on Ventilation Design and Control Technology of Zhongnanshan Extra-long Road Tunnel in fire mode. SW Jiaotong Univ. (2009) 
4. M. Wang, Q. Yang, Q. Zhao, P. Liu. Research on Disaster Prevention Plan of Qinling Zhongnanshan Ultra-long Highway Tunnel. Highway,11,87(2000)

5. G.LI, W. YI, B. LIU. Preliminary Analysis on Emergency Rescue in Erlangshan Tunnel of SichuanTibet Road. Fire Sci. Technol. 06,806(2006)

6. Q. Zhou, X. Zhou. Research on Fire Prevention and Rescue for Yanmenjie Tunnel. Fire Sci.Technol. 28,599. (2009)

7. JTG/J D70/2-01-2014, Code for Design of Ventilation of Road Tunnel. China Communications Press,(2014) 